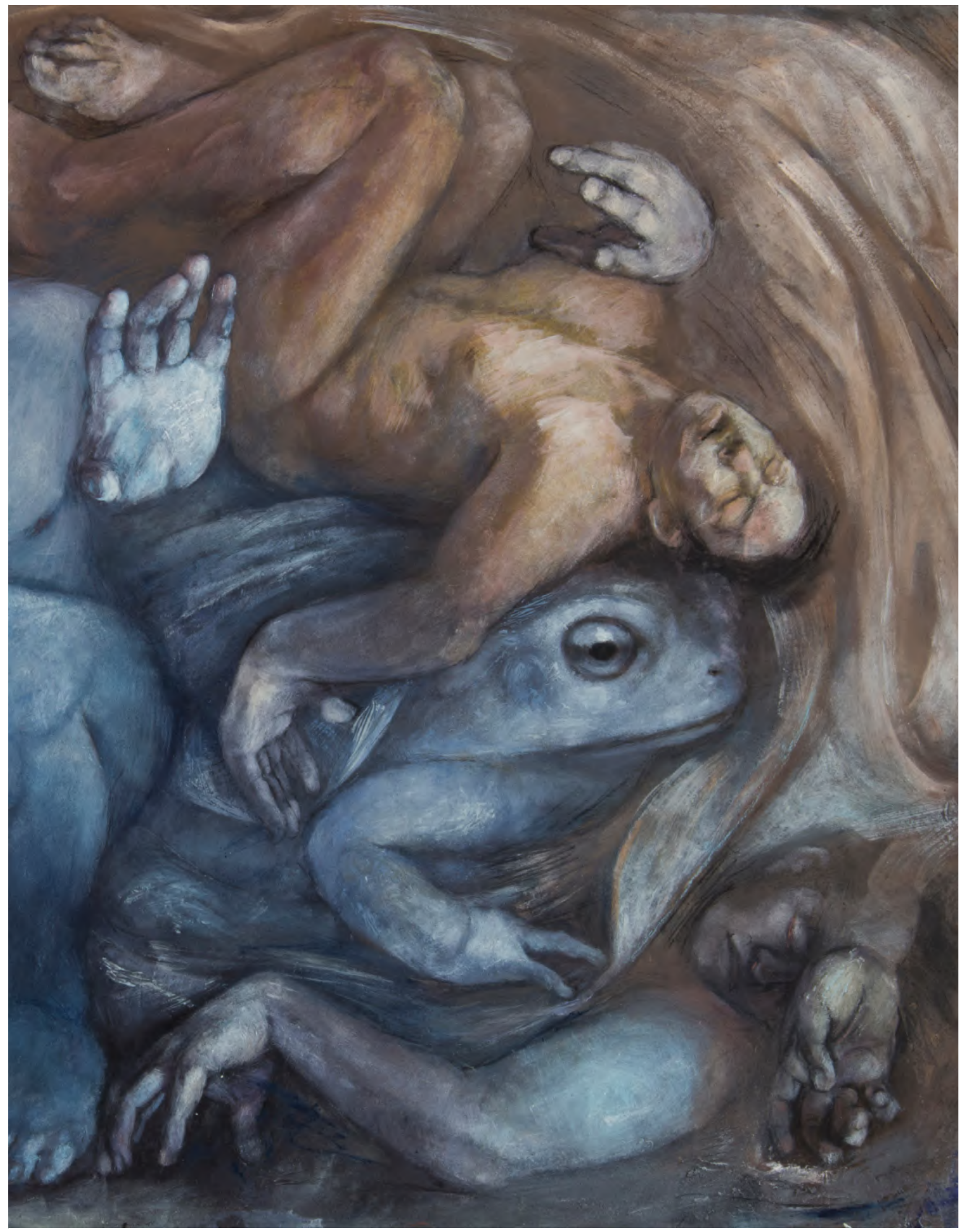

PESADILLA | Temple sobre tela y madera $\mid 70 \times 100 \mathrm{cms}$ 


\section{REFERENTES Y REPRESENTACIONES SIMBÓLICAS DE ESTUDIANTES ANTE EL ESTUDIO A DISTANCIA POR GONFINAMIENTO POR GOVID-19}

\section{REFERENCES AND SYMBOLIC REPRESENTATIONS OF STUDENTS IN THE DISTANCE STUDY BY COVID-19 CONFINEMENT}

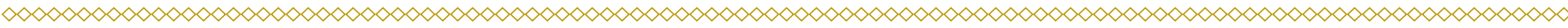

TERESA DE LA 0 DE LA 0

https://orcid.org/0000-0001-6837-9801 RIGOBERTO GARCÍA CUPIL

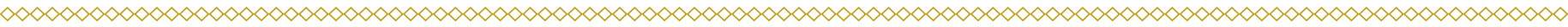

RESUMEN

Durante generaciones la educación a distancia ha dado cuenta de su pertinencia, calidad, contribución y posibilidades. De acuerdo al momento histórico, ésta diversificó sus alcances y métodos de enseñanza, existiendo con la premisa de formar y ofrecer mecanismos viables que atendieran las condiciones y características de sus usuarios, por lo que su pertinencia y calidad no son referentes espontáneos que intenten agudizarse en la actualidad. Consecuente y en resultado al confinamiento por COVID-19, tanto gobiernos como instituciones educativas, tal fue el caso de la Universidad Juárez Autónoma de Tabasco, implementaron como estrategia emergente a la educación virtual para continuar con sus funciones sustantivas, de ahí la relevancia de este estudio que aborda la percepción e implicaciones presentes en los estudiantes de la División Académica de Educación y Artes durante el ciclo escolar Febrero-Agosto de 2020.
ABSTRACT

For generations, distance education has realized its relevance, quality, contribution and possibilities. According to the historical moment, it diversified its scope and teaching methods, existing with the premise of training and offering viable mechanisms that would meet the conditions and characteristics of its users, so its relevance and quality are not spontaneous references that try to sharpen it today. Consequently and as a result of the confinement by COVID-19, both governments and educational institutions, such was the case of the Autonomous University of Tabasco, implemented virtual education as an emerging strategy to continue with its substantive functions; the relevance of this study that addresses the perception and implications present in the students of the Academic Division of Education and Arts during the February-August 2020 school year.

KEYWORDS: Education, Stress, Perceptions, Distance, Students, Virtual 
as condiciones actuales de salud han orientado a que los servicios, giros e instituciones diversifiquen sus actividades y subsistan en esquemas no convencionales como son los digitales. Tales condiciones han reivindicado el papel trascendente que la educación a distancia ha tenido en la formación y profesionalización de personas durante varias generaciones.

Los esquemas formativos basados en la virtualidad han permitido que las Instituciones de Educación Superior (IES) continúen con sus funciones sustantivas relativas a su ejercicio y ofrezcan a los usuarios de los servicios la continuidad, medios, formación, desarrollo de competencias y conclusiones de ciclos escolares y de programas educativos.

Ante el establecimiento, reivindicación y auge que la educación a distancia ha tenido a nivel mundial como estrategia emergente por causa del confinamiento por COVID-19, las percepciones sobre su pertinencia, calidad, eficacia y trascendencia han sido amplias. Existen percepciones basadas en las subjetividades y

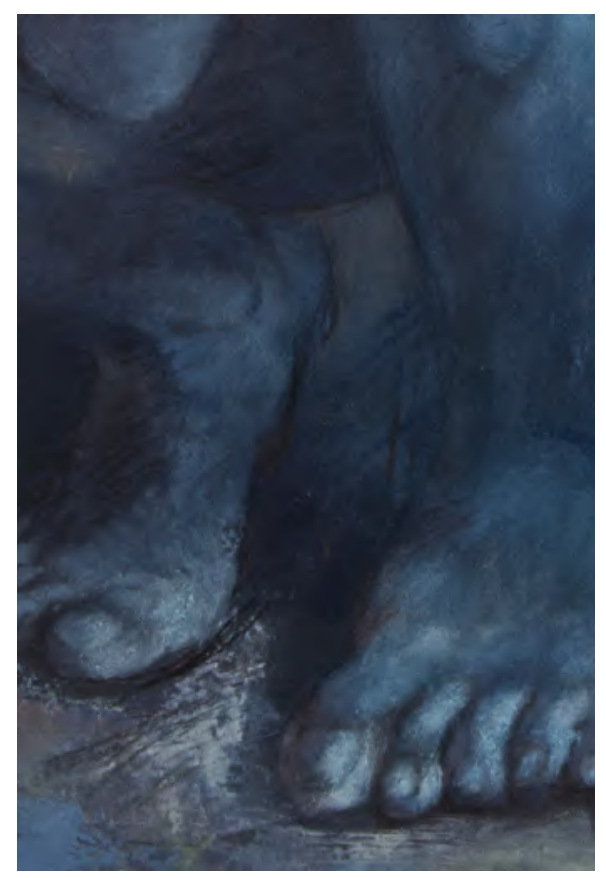

Presencia Universitaria

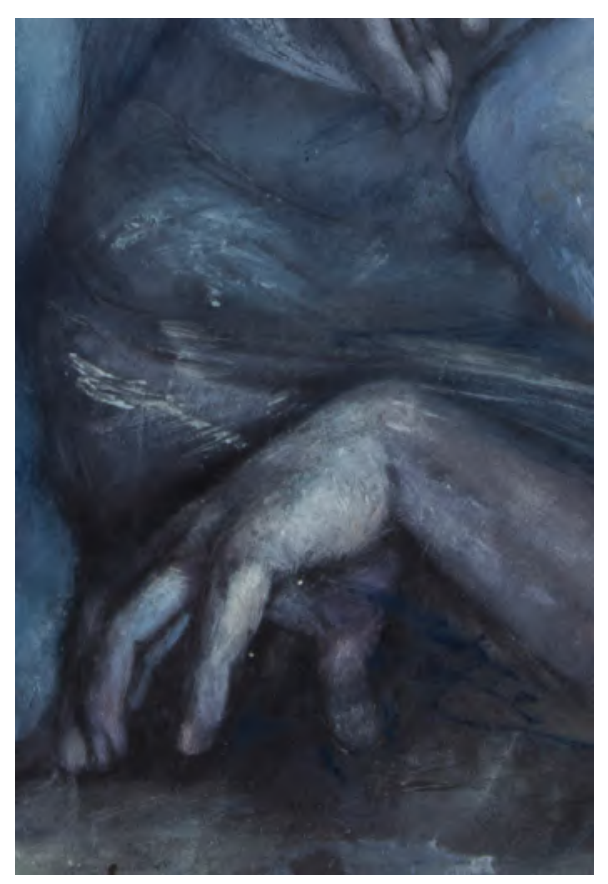

escasas experiencias de profesores y estudiantes, que en ocasiones son injustas y lejanas al verdadero valor y contribución que esta modalidad educativa continúa ofreciendo a todos.

No es de asombro que, tanto los órdenes de gobiernos, las instituciones, los profesores, los padres de familia y los estudiantes, tengan durante el confinamiento por COVID-19 sus primeras experiencias como usuarios con la educación a distancia, las comunidades virtuales de aprendizaje, el aprendizaje cooperativo, la escuela en casa y el auge de una cultura del maker. Estas y otras pedagogías tienen un gran potencial para facilitar el aprendizaje a distancia, tal como se ha hecho notar durante esta pandemia, por ello, es importante precisar que sus alcances siempre estarán determinados por el nivel de compromiso, dedicación, autogestión y motivación de los agentes que participen.

\section{PLANTEAMIENTO DEL PROBLEMA}

Ante los retos iniciales que supuso para el sistema educativo mexicano y las IES de la nación, el continuar ofreciendo

72 sus servicios de manera virtual, pueden enlistarse ejemplos oportunos sobre la adopción de estas pedagogías no convencionales y emergentes, tal como es el caso de la Secretaría de Educación Pública (SEP) quien, a través de las acciones emprendidas en las distintas regiones del país, mitigó los efectos de la propagación del virus COVID-19, al decretar el 23 de marzo de 2020 la suspensión total de actividades administrativas y académica de manera presencial en todos los niveles educativos del país.

Producto de las medidas establecidas por la SEP y de las directrices otorgadas por el Fondo de las Naciones Unidas para la Infancia (UNICEF) y la Organización de las Naciones Unidas para la Educación, la Ciencia y la Cultura (UNESCO) (2020), se permitió el impulso hacia lo digital, con el Programa Aprende en Casa. La pandemia obligó a diseñar una estrategia equitativa de educación a distancia para no suspender el aprendizaje de niñas, niños, adolescentes y jóvenes, y mantener la continuidad del ciclo escolar 20192020, cuyos contenidos llevaban el 73 por ciento de avance (SEP, 2020).

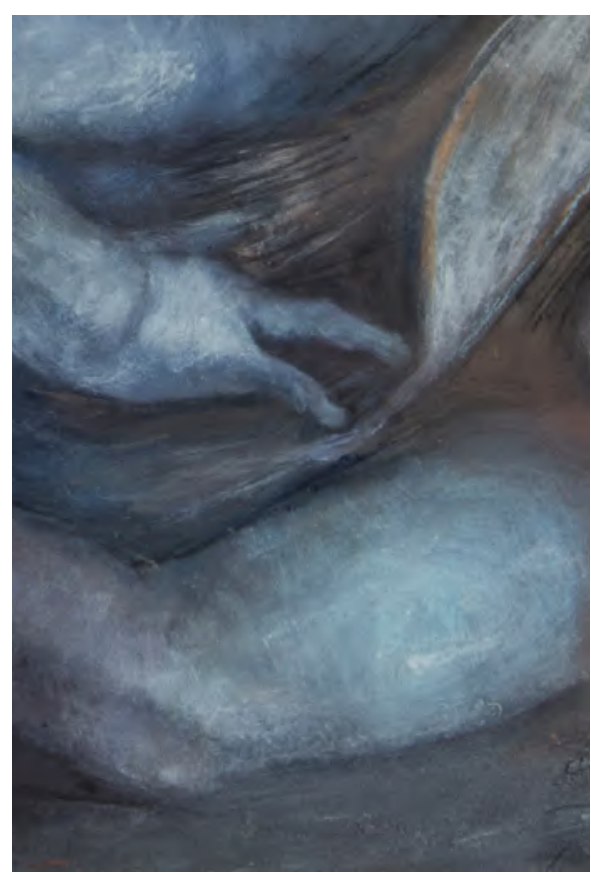


Por su parte, un sin número de universidades del país establecieron convenios de capacitación, colaboración y uso con Microsoft para habilitar a administrativos, profesores y estudiantes en el manejo de la Plataforma Teams. Con la premisa fundamental de integrar aulas de clases virtuales colaborativas, conectar en comunidades profesionales de aprendizaje, administrar el aprendizaje remoto e impulsar entre la comunidad académica la colaboración, comunicación y personalización con la plataforma.

De las condiciones expuestas y por las iniciativas pertinentes realizadas por la Dirección de Tecnologías de Información e Innovación de la Universidad Juárez Autónoma de Tabasco (UJAT, 2020), se pone en uso para toda la comunidad académica y administrativa de la Universidad el acceso al Aula Virtual UJAT, que mediante la Plataforma Teams se solventa la atención de servicios a la comunidad y procesos de enseñanza-aprendizaje correspondientes al ciclo escolar Febrero-Agosto 2020.

Los cambios radicales de la forma de enseñar, de aprender e incluso de vivir consecuentes por los efectos de una pandemia, son notorios en todos y experimentado en diversos sentidos por parte de profesores y estudiantes. A partir de ahí el interés de este trabajo de inves-

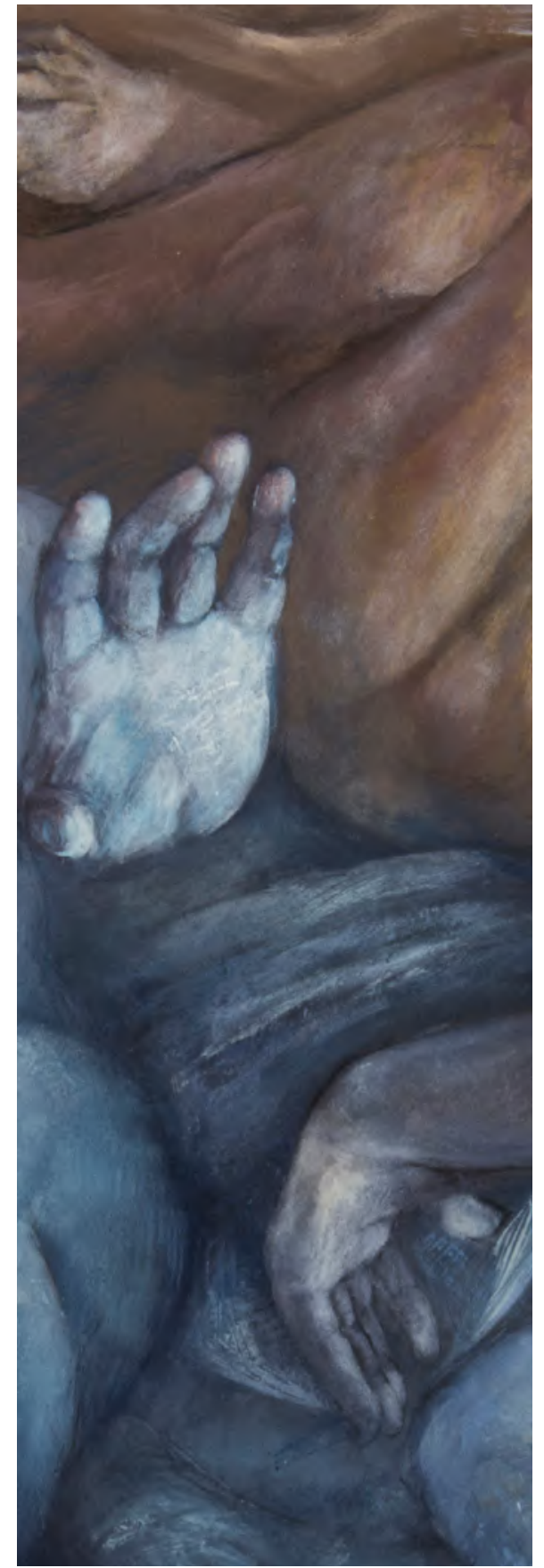

tigación, el cual pretendió conocer la opinión que los alumnos de la División Académica de Educación y Artes (DAEA) de la UJAT tienen sobre la estrategia emergente de uso de la educación a distancia durante el ciclo escolar vigente ante el confinamiento por la pandemia del COVID-19.

\section{OBJETIVO GENERAL DE LA INVESTIGACIÓN}

Conocer la opinión que los alumnos de la División Académica de Educación y Artes (DAEA) de la UJAT tienen sobre la estrategia emergente de uso de la educación a distancia durante el ciclo escolar vigente ante el confinamiento por la pandemia del COVID-19.

\section{OBJETIVOS ESPECÍFICOS \\ DE LA INVESTIGACIÓN - HIPÓTESIS}

Las disposiciones de ánimos de los estudiantes son las que determinan la aceptación, logro e impacto de la estrategia emergente institucional (UJAT) para el estudio a distancia ante el confinamiento provocado por la pandemia COVID-19.

\section{JUSTIFICACIÓN \\ DE LA INVESTIGACIÓN}

En esta nueva sociedad que exige una renovación constante del conocimiento y una mayor fluidez de los procesos educativos basados en la tecnología, converge

\section{Este trabajo pretendió conocer la opinión que los alumnos de la División Académica de Educación y Artes (DAEA) de la UJAT tienen sobre la estrategia emergente de uso de la educación a distancia durante el ciclo escolar vigente ante el confinamiento por la pandemia del COVID-19.}




\section{Converge el señalamiento de García Aretio (1999) al llamar a las épocas venideras, hoy en día las actuales, como una ruptura de paradigmas a través del surgimiento y auge de una nueva visión por medio de la educación virtual.}

el señalamiento de García Aretio (1999) al llamar a las épocas venideras, hoy en día las actuales, como una ruptura de paradigmas a través del surgimiento $y$ auge de una nueva visión por medio de la educación virtual.

Para responder a estas exigencias y al cumplimiento de los objetivos de proceso de enseñanza-aprendizaje, a través de la mediación tecnológica, se hace posible disponer de las fuentes de información (inaccesibles en otros tiempos, provenientes de diversos lugares del mundo, muy alejados entre sí) y que aparecen como resultado del desarrollo acelerado de la informática, de la telemática, de los multimedios y del internet. La actualización en el uso de las TIC en el sector educativo, se constituye en la actualidad en una competencia básica de cualquier profesional, y no en una tarea adquisitiva casi exclusiva de docentes y alumnos coincidentes en procesos de formación profesional.

\section{MÉTODO - SELECCIÓN \\ DE LA MUESTRA (DESCRIPCIÓN)}

Se observó que del total de 3,821 estudiantes matriculados en la DAEA de la UJAT, participaron 758 en la resolución del formulario, de los cuales 703 corresponden a los diversos programas educativos de la División Académica y el restante, a otras de la misma institución.

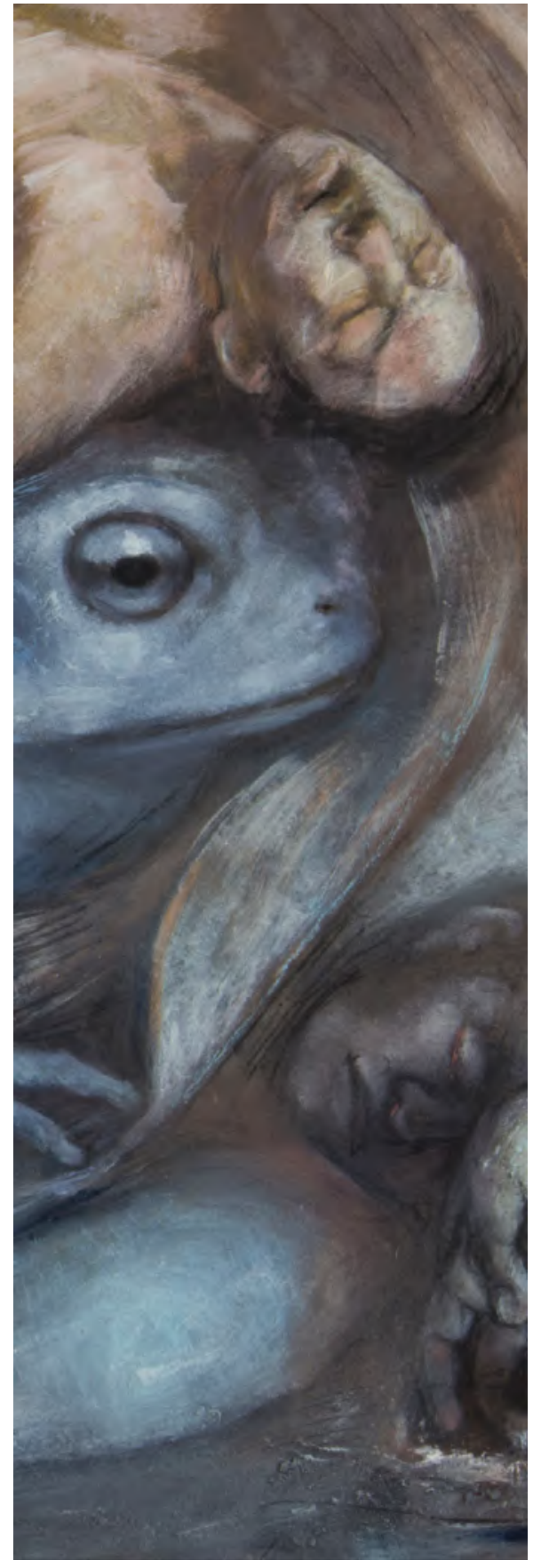

\section{INSTRUMENTO} DE INVESTIGACIÓN

El trabajo Percepciones de los estudiantes sobre la estrategia emergente Aula Virtual de la UJAT durante el confinamiento por COVID-19 retoma las acepciones anteriormente citadas y enfoca sus esfuerzos en un diseño metodológico que permita conocer las percepciones e implicaciones sobre el estudio a distancia durante el confinamiento. Para ello, se instrumentó un formulario en línea que abordó diversas dimensiones de ánimo; estrés; competencias y percepciones posibles a experimentar.

\section{PROCEDIMIENTO}

El trabajo en cuestión se desarrolló bajo un enfoque mixto con el empleo de 2 métodos para obtener y ampliar el análisis de la información sobre el problema. Se combinaron instrumentos de ambos enfoques para generar mayor amplitud en el proceso de recolección de datos. Partió de la observación participante y no participante realizadas durante el inicio del proceso de incursión en el estudio a distancia. Las reflexiones iniciales obtenidas fueran categorizadas y ampliadas a través de un formulario en Google, con el propósito de usar la recolección y análisis de datos para resolver cuestionamientos propios del estudio de investigación y probar la hipótesis establecida previamente; confió en la 
medición numérica, en el conteo y en el uso de la estadística para establecer con exactitud patrones de comportamiento en una población.

\section{RESULTADOS}

Se observó que de 3,821 estudiantes matriculados en la DAEA de la UJAT, participaron 758 en la resolución del formulario, de los cuales 703 corresponden a los diversos programas educativos de la División Académica y el restante, a otras de la misma institución.

Poder dimensionar el análisis de las experiencias y percepciones que los alumnos viven al cursar un ciclo escolar a distancia, es otra de las pretensiones de este trabajo, pues es importante abordar cómo esta estrategia puede impactar en la formación profesional, considerando la importancia actual que las TIC tienen en el sector educativo.

La educación virtual es la modalidad que eleva la calidad de la enseñanza-apren- dizaje al respetar su flexibilidad o disponibilidad, es decir, se puede canalizar para tiempos y espacios variables. Beneficia al estudiante al tener la libertad de organizar sus tiempos de estudio para investigar y realizar las actividades asignadas por sus profesores.

Los elementos estadísticos que precisan los sujetos objetos de este trabajo aluden a los estudiantes activos, matriculados y pertenecientes a los diversos ciclos escolares en la DAEA. 754 alumnos resolvieron el instrumento, de los cuales:

- 287 son alumnos de la licenciatura en Ciencias de la Educación.

- 340 alumnos de la licenciatura en Idiomas.

- 53 alumnos de la licenciatura en Comunicación.

- 16 alumnos de la licenciatura en Gestión y Promoción de la Cultura.

- 3 alumnos del Técnico Superior Universitario en Música.

- 52 alumnos de otras Divisiones Académicas que sin indicación y de forma voluntaria dieron respuesta al instrumento.

El enlace fue difundido en redes sociales; el proceso de recolección de datos comprendió del 22 al 30 de marzo de 2020 y se obtuvo a través de la instrumentación en línea del formulario y dispuesto en la red social Facebook.

De acuerdo a lo citado anteriormente, los siguientes puntos exponen datos relevantes de este trabajo: pretende saber la opinión de los alumnos respecto al proceso de cursar un ciclo escolar con mayor actividad en línea. Se pudo conocer cómo fue su experiencia; identificar cuáles fueron las debilidades que tuvieron los alumnos de la DAEA para cursar este ciclo escolar bajo la modalidad de educación a distancia. Se expone el nivel de preparación de profesores y alumnos con respecto al área tecnológica y en educación a distancia. Se identifican las habilidades que desarrollaron los alumnos como parte de su formación

\section{Gráfica 1. Disposición de recursos para el estudio a distancia}

\section{Percepciones de los estudiantes}

¿Cuentas con los medios, recursos didácticos, tecnológicos y de internet para el estudio a distancia?

758 respuestas

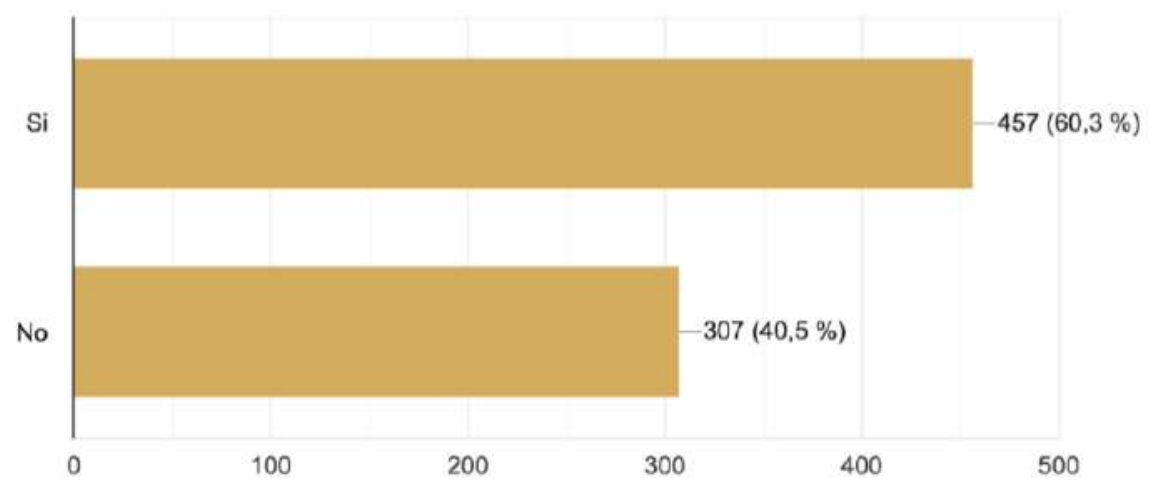




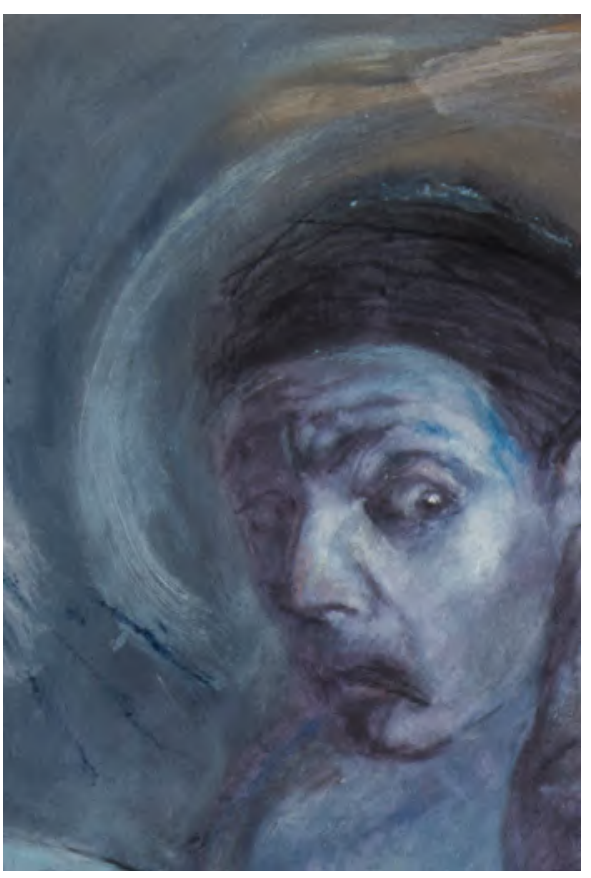

profesional. Se conocen las deficiencias que pudieron haber impedido al alumno no tener un proceso exitoso al cursar el ciclo escolar mediante la educación a distancia. Se incluyen algunas explicaciones sobre el sentir respecto al desempeño de sus profesores en este proceso y se analizan los resultados del proceso de educación a distancia que fue implementado para hacer frente a la contingencia del COVID-19. De esta manera conoce cómo es la cultura que tienen los alumnos de la DAEA respecto a la educación a distancia, y cómo pueden desenvolverse frente a una situación de emergencia.

Es importante el papel que las Tecnologías de la Información y las Comunicaciones en el aprendizaje por el número de sentidos que pueden estimular, y la potencialidad de los mismos en la retención de la información. Diversos estudios, ya clásicos, han puesto de manifiesto que se recuerda el $10 \%$ de lo que se ve, el $20 \%$ de lo que se oye, el $50 \%$ de lo que se ve y oye, y el $80 \%$ de lo que se ve, oye y hace. Algunas de las TIC son idóneas para propiciar la retención de la información; los multimedia orientan

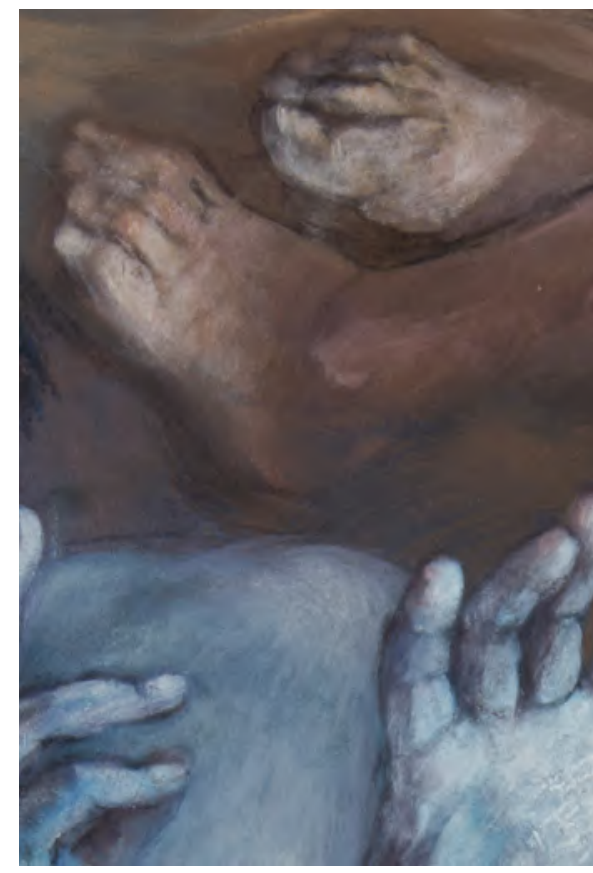

a diversos estilos de aprendizaje, y los interactivos, donde el alumno además de recibir la información por diferentes códigos tiene que realizar actividades (Gráficas 2 y 3).

La pertinencia y eficacia de las modalidades no convencionales tal como lo es la educación a distancia estarán supeditadas al nivel de motivación, compromiso, disposición, disciplina y autocontrol de los alumnos y docentes, como elementos importantes para determinar su impacto y trascendencia.

Se observa que las percepciones negativas y el rechazo de los estudiantes sobre la implementación del Aula Virtual UJAT radica en el bajo nivel de compromiso para la realización de actividades, tal como se visualiza en la Gráica 4.

La motivación, desde la perspectiva de los motivos, alude a algo mucho más complejo. No se pueden obviar las condicionantes y obstáculos de carácter políticos, sociales, económicos, geográficos, distinguiéndose como un punto importante a tener en cuenta en el estu-

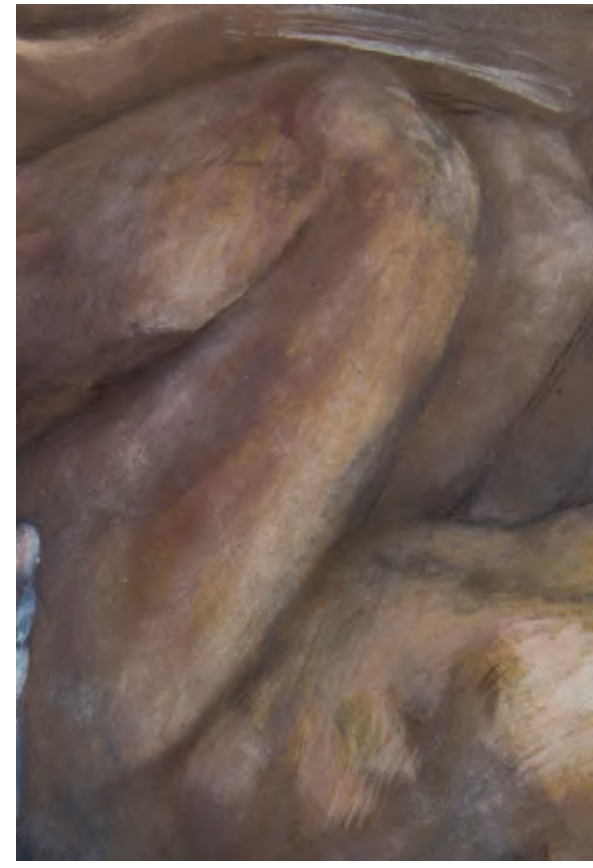

dio a distancia y esto es, lo afectivo, en donde los sentimientos, motivaciones, la recuperación de la motivación inicial son determinantes para los logros de los propósitos de cualquier curso. Situación carente en los estudiantes de la DAEA para reconocer que las condiciones actuales de salud obligan al confinamiento y que la opción viable para el estudio es el esquema a distancia. A pesar de ello, existe un alto porcentaje de insatisfacción por el estudio (Gráfica 5).

El estudio en cuestión aborda las implicaciones determinantes en la aceptación de una estrategia institucional al establecer esquemas de formación en la virtualidad ante el confinamiento por COVID-19. De los datos sobresalientes e identificados en este trabajo radica en que dichas percepciones están sujetas a factores asociados con la falta de experiencia, falta de recursos para la conectividad, proactividad para el afrontamiento de situaciones nuevas, el manejo de emociones y la ausencia de estrategias metacognitivas que le permitan al estudiante la autogestión, la disciplina y el trabajo autodidacta. Percibiendo este 


\section{Gráica 2. Dominio tecnológico para el uso de herramientas y plataforma}

Con respecto al dominio tecnológico para el uso de herramientas y de la plataforma ¿Cómo valoras tus conocimientos?

758 respuestas

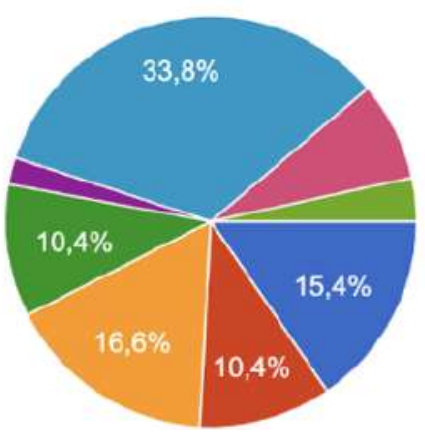

Excelente

Muy bueno

Bueno

Regular

Insuficiente

Bien

En desacuerdo

$\mathrm{Mal}$

Gráfica 3. Plataforma de mayor uso durante el confinamiento por COVID-19

¿Cuál es la plataforma/herramienta que más usan para el estudio a distancia?

758 respuestas

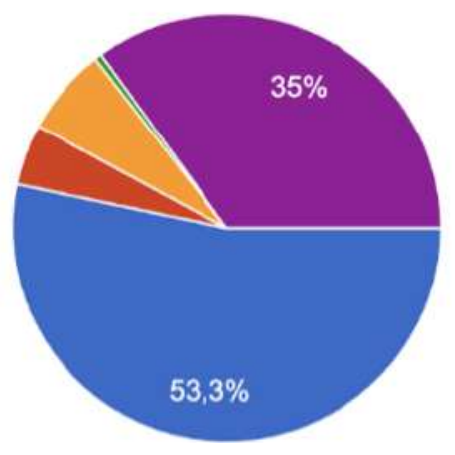

Microsoft Team

Edmodo

Zoom

Moodle

Redes sociales y sistemas de mensajería 


\section{Gráfica 4. Frecuencia para la realización de actividades}

¿Con qué frecuencia realizas las actividades señaladas para este periodo de contingencia en modalidad a distancia?

758 respuestas

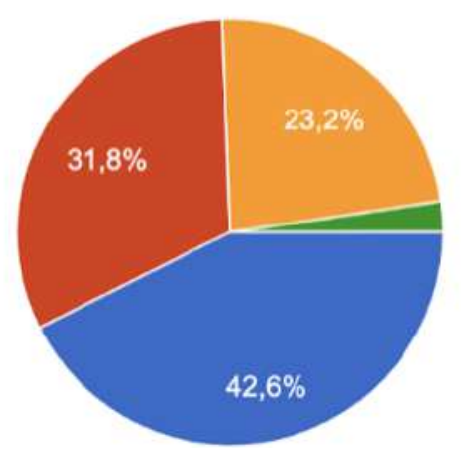

Siempre

Casi siempre

$A$ veces

Nunca

\section{Gráfica 5. Nivel de compromiso para el estudio a distancia}

¿Cómo te sientes con respecto a tu nivel de compromiso para el estudio a distancia durante esta contingencia?

758 respuestas

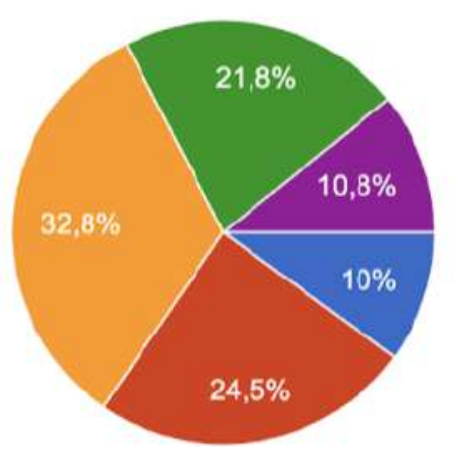

Muy satisfecho

Satisfecho

Medianamente satisfecho

Poco satisfecho

Insatisfecho 


\section{Gráfica 6. Disposiciones de ánimos por el estudio a distancia}

¿Con qué frecuencia experimentas fatiga, cansancio, sueño y dolor de cabeza por la carga de actividades señalada por tus profesores en modalidad a distancia?

758 respuestas

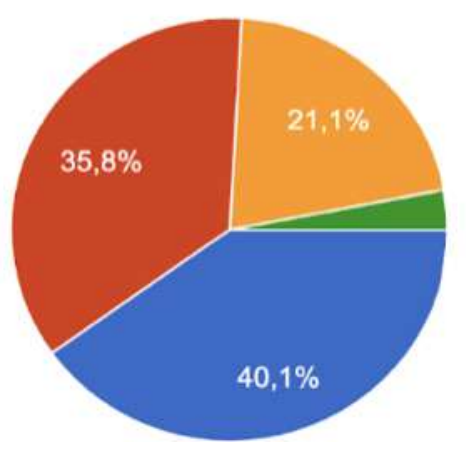

Nunca

Gráfica 7. Nivel de estrés en estudiantes de DAEA por el estudio a distancia

Si pudieras establecer el nivel de estrés que experimentas en estos momentos sobre el estudio a distancia ¿Cuál seria?

758 respuestas

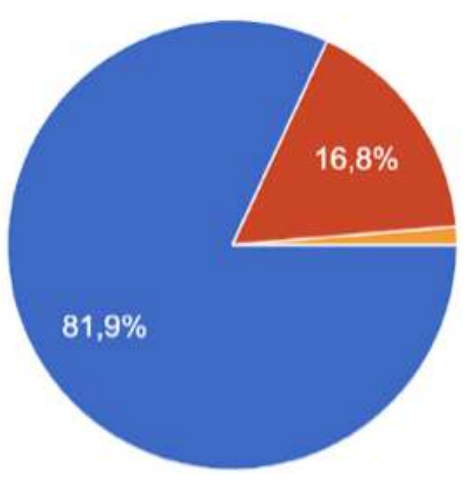

Mucho

Poco

Nada 


\section{Gráfica 8. Estrategias para el estudio a distancia}

¿Con qué frecuencia te preparas asertivamente mediante apuntes, comunicación permanente con compañeros - profesor, lecturas, análisis, uso eficiente de la tecnologia, etc. para hacer del estudio a distancia una experiencia favorable?

758 respuestas

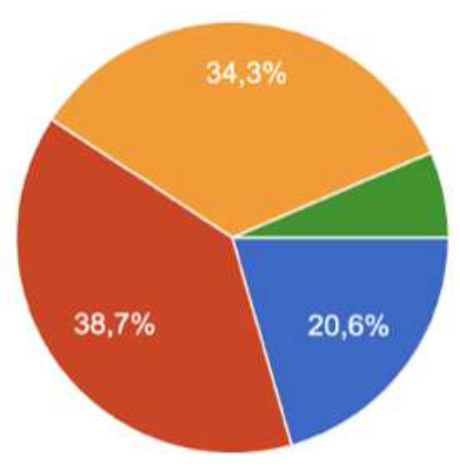

Siempre

Casi siempre

A veces

Nunca

\section{Gráfica 9. Expectativas de aprendizaje de un sistema a otro}

¿En qué nivel consideras serán tus aprendizajes en plataforma a diferencia de clases presenciales?

758 respuestas

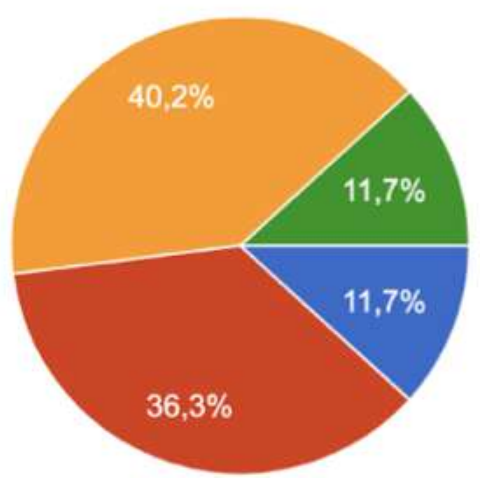

Suficiente

Medianamente satisfecho

Poco

Nulo 


\section{Gráfica 10. Experiencia en la modalidad a distancia}

¿Cómo valoras tu experiencia de estudio en modalidad a distancia durante esta contingencia?

758 respuestas

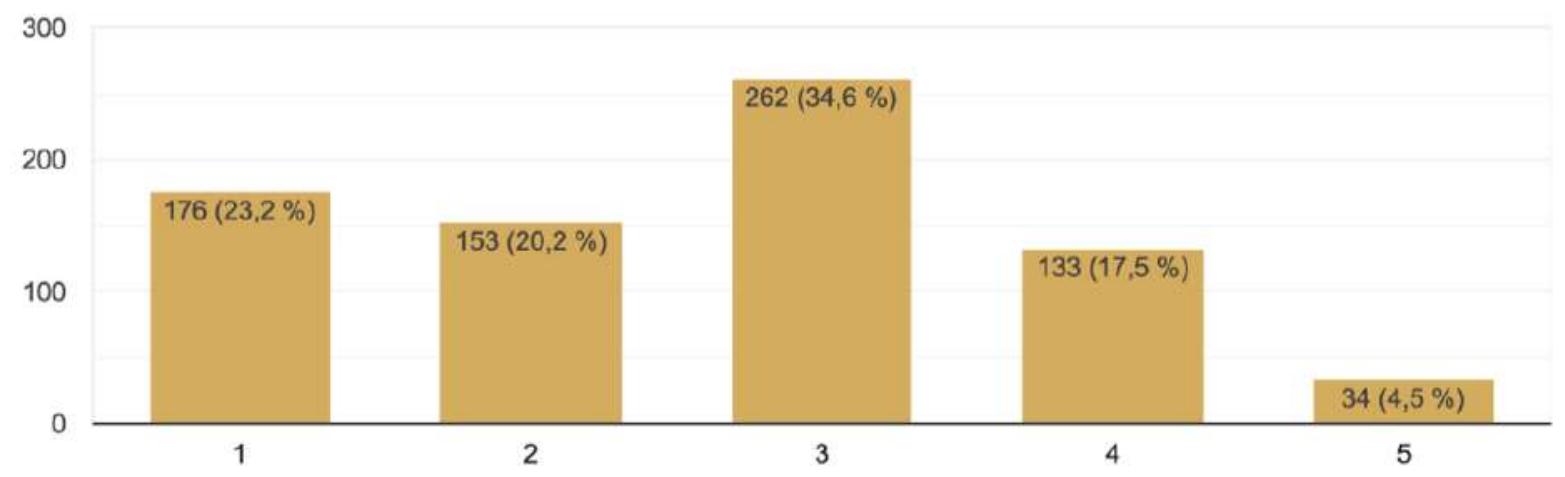

\section{Gráfica 11. Desempeño de profesores}

¿Cómo te sientes con respecto al desempeño de tus profesores durante esta contingencia?

758 respuestas

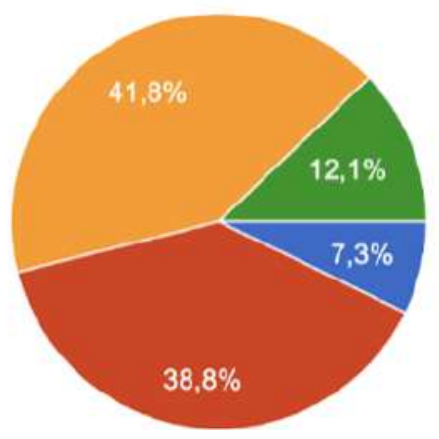

Muy satisfecho

Satisfecho

Poco satisfecho

Insatisfecho 


\section{Gráfica 12. Dominio tecnológico de profesores}

\section{¿Cómo consideras de tus profesores el dominio tecnológico de los recursos y plataforma?}

758 respuestas

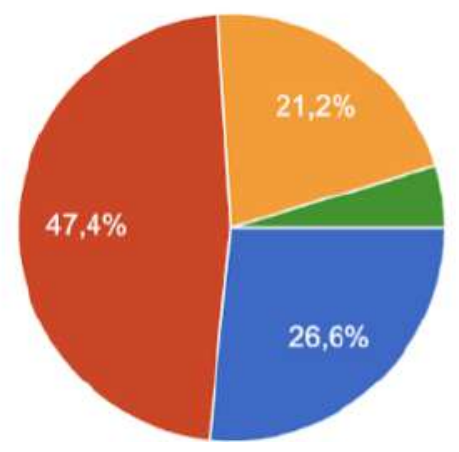

hecho como referente de estrés, fatiga, cansancio y malestar físico (Gráfica 6).

El estrés, las estrategias de afrontamiento y las emociones han sido ampliamente estudiadas en la Psicología desde diferentes perspectivas. Los 3 elementos citados como variables psicológicas son explicadas por el Modelo Transaccional. Este modelo concibe la presencia de estrés como la interacción entre el individuo y su entorno, además supone que no es el evento ambiental aislado 0 la respuesta lo que define al estrés, sino la apreciación que el individuo tiene de la situación, la cual es valorada en dos niveles: valoración primaria determina hasta qué punto la relación con el ambiente es de daño/pérdida, de amenaza, de desafío o de beneficio, el conjunto de ellas conducen a la percepción de estrés.

En caso de que el individuo evalúe la relación como irrelevante 0 benigna, no se percibe estrés ni implicaciones aversivas en su vida. Si sucede lo contrario, el individuo se exige la movilización para afrontar la situación, lo cual corresponde a la valoración secundaria, en donde analiza con qué recursos de afrontamiento dispone y la posibilidad de utilizar una estrategia efectiva ante dicha situación, en este caso ante el estudio a distancia propuesto por la UJAT en respuesta al confinamiento por COVID-19.

De acuerdo al autor expuesto para el abordaje del Modelo Transaccional, las valoraciones primarias como secundarias interactúan para determinar el grado de estrés percibido y la reacción emocional, este último propuesto por Lazarus en el 2000 y que, en relación al tema bajo estudio, se identifica que el $81 \%$ de alumnos encuestados presentan altos niveles de estrés resultado del estudio en modalidad a distancia (Gráfica 7).

Desde otra perspectiva de análisis, en el ámbito educativo se le denomina disposiciones de ánimo, a aquellas situaciones que generan los mismos efectos benignos/adversos en los que participan en el proceso formativo y ejercen una influencia positiva/negativa y de tensión a la salud física y mental. Por ello, al momento del establecimiento del ciclo escolar febrero-agosto 2020 por parte
Suficiente

Medianamente satisfecho

Poco

Nulo de la UJAT en modalidad virtual generó poca aceptación y esto es resultado de los recursos psicológicos limitados con los que dispone el profesorado y los estudiantes, donde las estrategias de afrontamiento son carentes, y, por lo tanto, los esfuerzos cognitivos y conductuales fueron durante el ciclo escolar constantemente cambiantes. Situación que se identifica en la percepción con respecto a estrategias para hacer favorable la experiencia, en las valoraciones sobre el desempeño de los profesores (Gráficas 8, 9 y 10).

La modificación de los entornos de enseñanza-aprendizaje, como consecuencia del uso de las TIC, implica, a su vez, un cambio en las formas en las que se lleva a cabo la enseñanza. McDonald y Reushle (2002) plantean que uno de los principales cambios 0 modificaciones ocurre en la filosofía de enseñanza que cada profesor tiene 0 ha desarrollado a lo largo de su trayectoria docente. Las formas de interacción con los alumnos, las expectativas tanto del profesor como de los alumnos, y los patrones de trabajo y actividades, se configuran de manera 


\section{Cossetti (2000) señala que el conocimiento y las habilidades que posee el profesor para impartir cursos tradicionales resultan insuficientes cuando se trata de procesos de enseñanza aprendizaje en modalidades virtuales.}

diferente. Las labores y tareas que desempeña el profesor, por consecuencia, se diversifican y se complementan con la interiorización de diversos roles.

Cossetti (2000) señala que el conocimiento y las habilidades que posee el profesor para impartir cursos tradicionales resultan insuficientes cuando se trata de procesos de enseñanza aprendizaje en modalidades virtuales. La efectividad y calidad de los procesos formativos en modalidad a distancia son corresponsables con la figura del profesor, el cual puede ser nombrado como asesor, tutor, instructor, facilitador, o de algún otro modo tipificable de acuerdo a las teorías existentes.

Lo relevante aquí es el papel que jugó la planta académica de la DAEA durante el ciclo escolar febrero-agosto 2020 y que, en términos de apreciación de los estudiantes, consideran sentirse con experiencias negativas con respecto al desempeño de los mismos, en razón que $53.9 \%$ los valoran como poco satisfecho e insatisfecho (Gráficas 11 y 12).

\section{CONCLUSIONES}

Los efectos de una pandemia sin importar la época histórica y sociedad a la que se pertenezca ejercerán en todos, cambios radicales que van desde las formas de percibir la propia vida hasta la modifi- cación en que se realizan las actividades habituales, laborales, sentimentales y fraternales.

La consecuencia, impacto y factores asociados a cada grupo humano estarán siempre sujetas al nivel de previsión, provisión y recursos con los que se cuente para hacer frente a la inestabilidad económica, emocional y social que se experimentará como parte del día a día. De ahí que sería muy injusto juzgar desde la particularidad de la comunidad de profesores y estudiantes la pertinencia 0 no de una estrategia institucional como ha sido la implementación a distancia del ciclo escolar febrero-agosto 2020. Esto no excluye a la institución de la atención de la necesidad identificada para la habilitación en materia de didáctica y tecnología para la educación a profesores, y el de dotar de estrategias de afrontamiento a estudiantes y al resto de la comunidad.

La educación y sus niveles, tal como se ha visto en nuestros contextos no ha tenido pausa, el gobierno del país y del estado han establecido estrategias para que las diversas instituciones de educación implementen, prueben e intenten compensar las desavenencias que trae consigo la pandemia y que pone a flote los verdaderos problemas que aquejan a los menos favorecidos. Ante esto, las críticas y el inicial rechazo por parte de estudiantes desacostumbrados al estudio independiente y de autogestión, puede ser nulo, pues pone de manifiesto mayor valor a las condiciones psicológicas de otras, e incluso, sobre las económicas (para la disposición de tecnologías).

Es indiscutible que la educación y sus procesos no son finitos, sino que para la UJAT requerirá establecer programas de capacitación y habilitación para profesores y estudiantes en temas relativos al manejo de emociones, al uso y trabajo en entornos virtuales de aprendizaje, en diseño instruccional y en el desarrollo de competencias tecnológicas para la enseñanza y el aprendizaje.

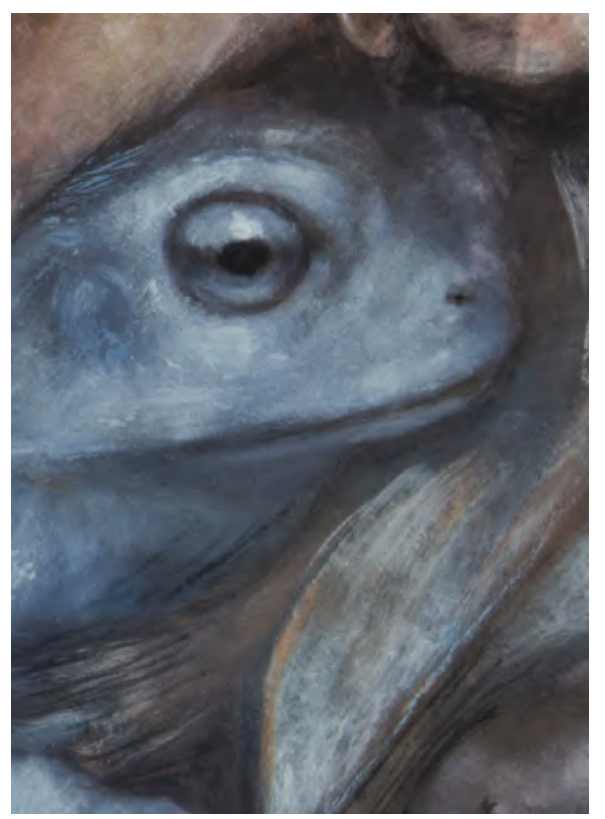




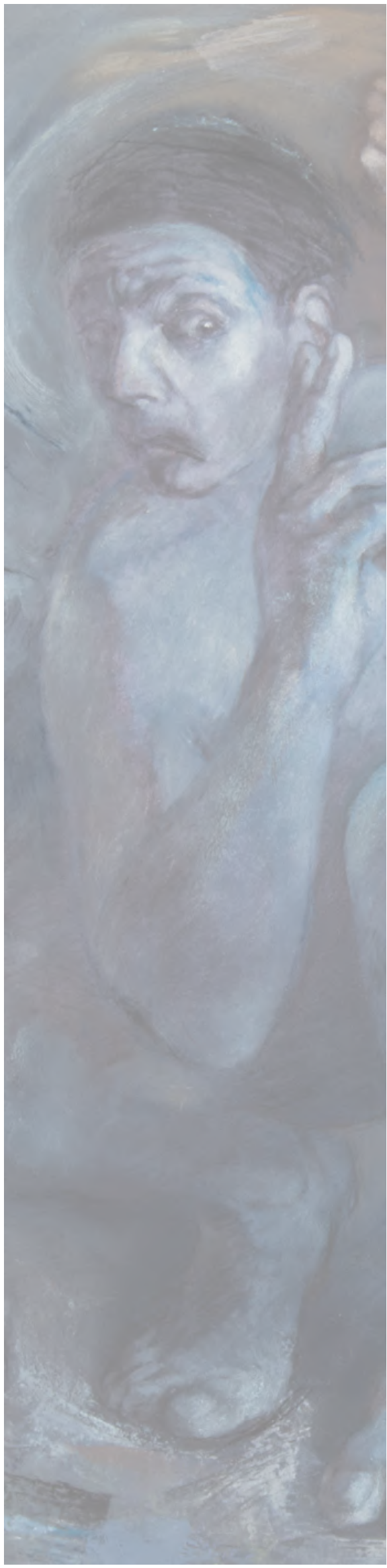

\section{REFERENCIAS}

Álvarez Valiente IB, Fuentes González HC. Didáctica del proceso de formación de los profesionales asistido por las tecnologías de la información y la comunicación. Revista Pedagogía Universitaria [revista en Internet]. 2005 [citado 11 Sep 2006];X(3):[aprox. 64p].

Chiecher, C. (2019). Estudiantes en contextos de educación a distancia. Variables vinculadas con el logro académico. RIED. Revista Iberoamericana de Educación a Distancia. En https://www.redalyc.org/jatsRepo/3314/331460297011/331460297011.pdf

Cosetti, A. (2002). Tutoring: A different background for the same role. Ed-Media 2002, Denver, AACE, (355-356).

Educación 3.0. (2020). Pedagogías emergentes en tiempos de confinamiento. EDUCACIÓN 3.0.

García Cabrero, B.; Pineda Ortega, V. J. (2011). Evaluar la Docencia en Línea: Retos y Complejidades. RIED. Revista Iberoamericana de Educación a Distancia, volumen 14, no 2, pp. $63-76$.

García, Aretio (2001). La educación a distancia. De la Teoría a la Práctica. Ariel. García, Aretio (2004). Barreras para aprendery educación a distancia. UNED. En https:/L www.researchgate.net/profile/Lorenzo_Garcia-Aretio2/publication/235664827_Barreras_para_aprender_y_educacion_a_distancia/links/02bfe51310314a1872000000/ Barreras-para-aprender-y-educacion-a-distancia.pdf

Gobierno de México (2020). Boletín №. 118 No se paralizó el Sistema Educativo ante la pandemia de COVID-19; regresará a clases fortalecido: Esteban Moctezuma Barragán. Gobierno de México. Disponible en https://www.gob.mx/sep/articulos/boletin-no-118-no-se-paralizo-el-sistema-educativo-ante-la-pandemia-de-covid-19-regresara-a-clases-fortalecido-esteban-moctezuma-barragan?idiom=es

Marchisio, Susana. Tecnología, educación y Nuevos Ambientes de Aprendizajes. Una revisión del campo y derivaciones para la capacitación docente en Revista RUEDA No. 5. La Rioja, EUDELAR, 2003, p. 17.

McDonald, J.; Reushle, S. (2002). Charting the role of the online teacher in higher education: winds of change. ASCILITE Conference 2002. Auckland, Nueva Zelanda.

UNAM (2017). Estrés, afrontamiento y emociones en estudiantes universitarios de sistema a distancia. Revista Electrónica de Psicología Iztacala. Disponible en http:// www.revistas.unam.mx/index.php/repi/article/view/60820/53671\# 


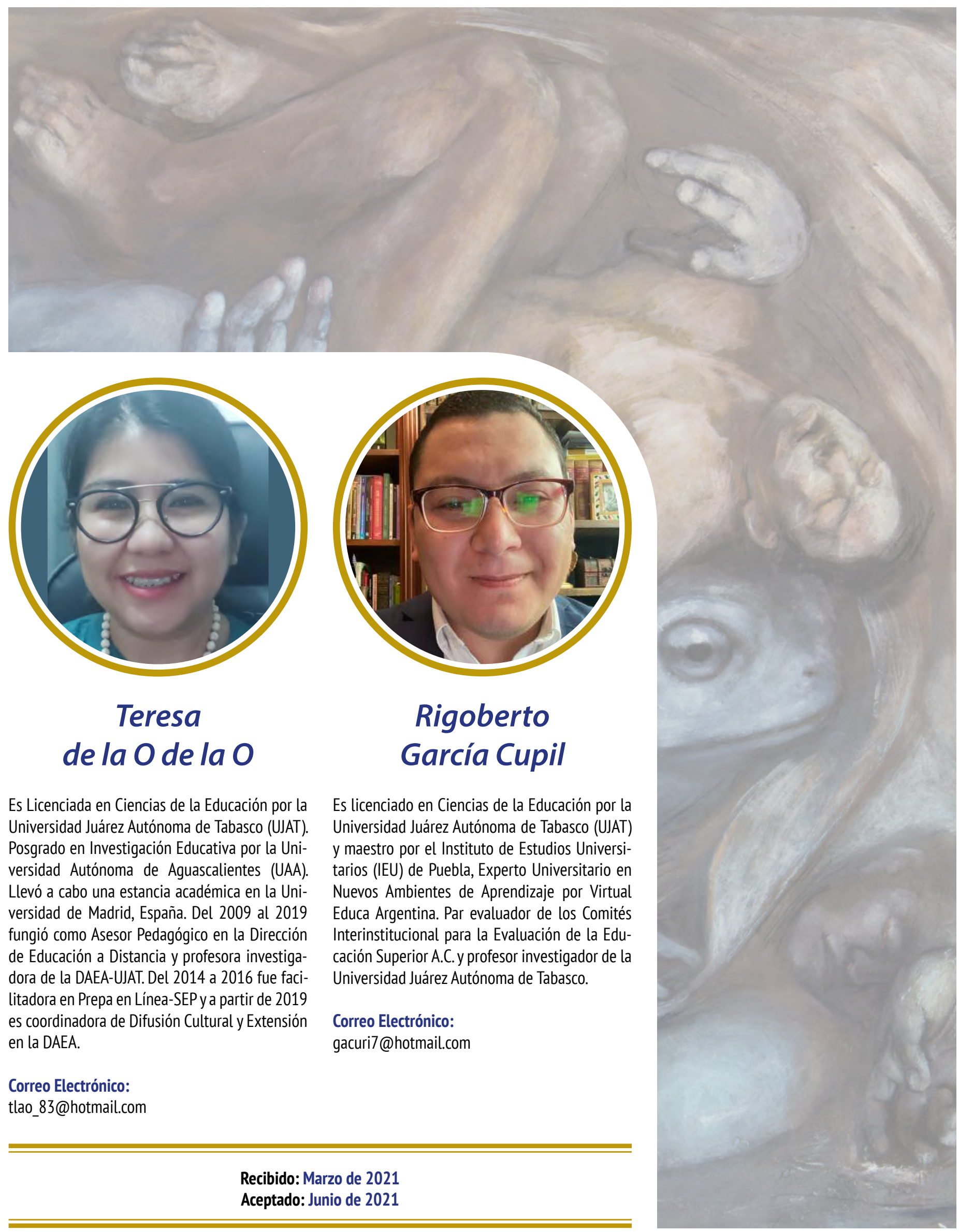




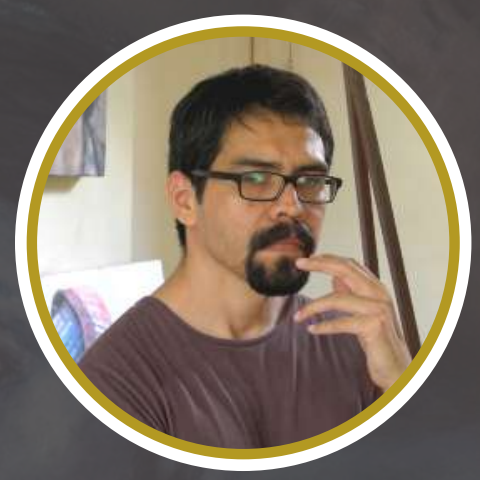

\section{Héctor Manuel Morales Orona}

Egresado de la Facultad de Artes Visuales de la Universidad Autónoma de Nuevo León. Inicia su profesión en el área de las artes gráficas, ilustrando en publicaciones de casas editoriales. Al mismo tiempo alterna esta actividad con la pintura mural. Después de este período inicial, su inquietud creativa se dirige al teatro, donde realiza estudios de arte dramático, cuyo aprendizaje originó nuevas reflexiones sobre el concepto de representación, y la experiencia del juego.

Más adelante, se dedica al estudio de las técnicas antiguas de la pintura haciéndolas su especialidad, y diversifica su profesión como pintor-dibujante y profesor de artes. Desde las técnicas antiguas y la importancia de los materiales, ha encontrado un campo nuevo de resignificaciones conceptuales, representando estas ideas sobre la materia, una fuerte línea de investigación que actualmente trabaja.

Cuenta con múltiples exposiciones colectivas e individuales. Ha participado en ediciones de la Reseña de la Plástica de Nuevo León y su obra se ha expuesto en México, Italia, Cuba y Canadá.

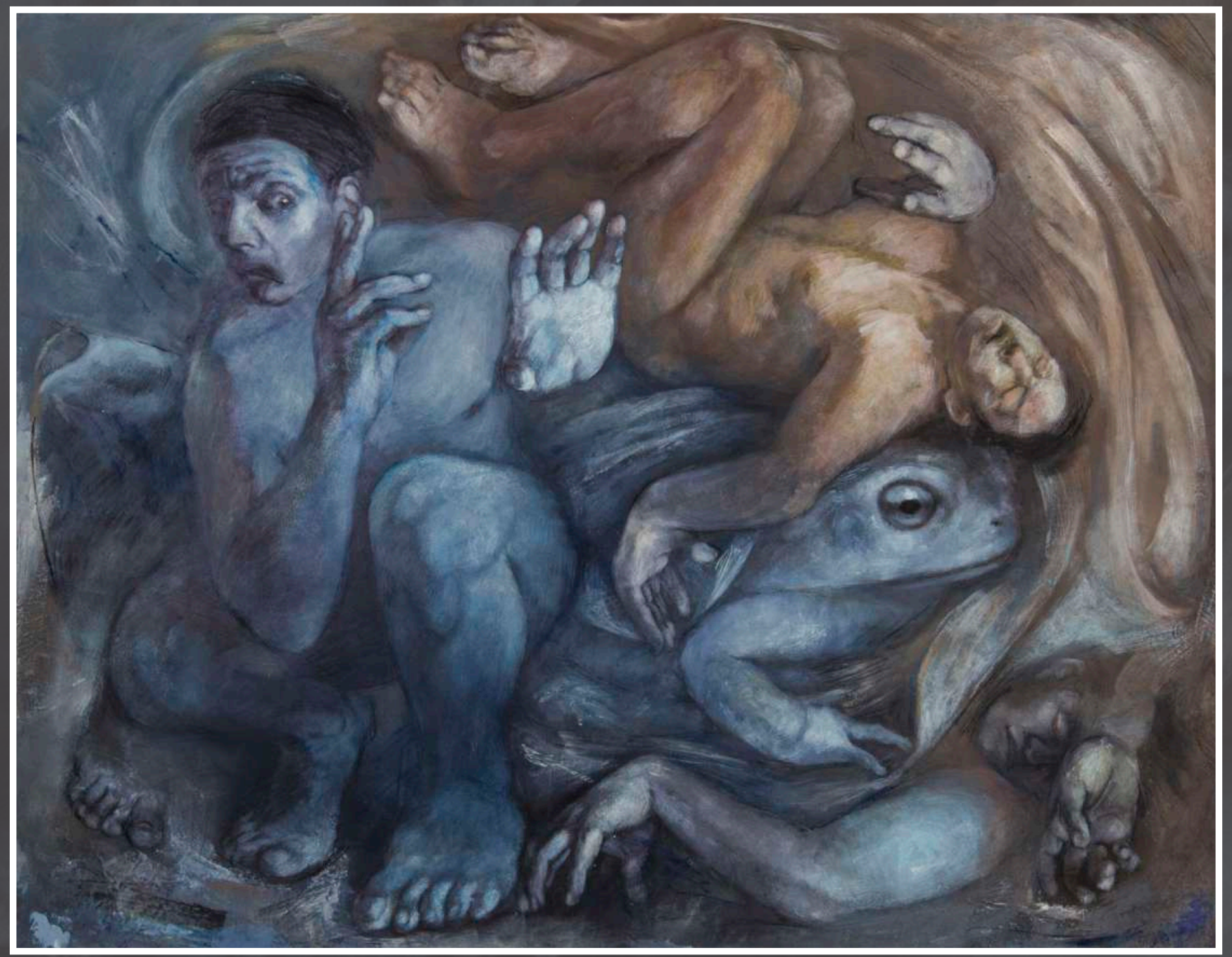

